\title{
Research and Design of vehicle Dynamic Weighing System Based on Piezoelectric Quartz Sensor
}

\author{
Jun Wei ${ }^{1, \mathrm{a}} \quad$ Mengjun Zhang ${ }^{1, \mathrm{~b}}$, Ligeng $\mathrm{Qin}^{2, \mathrm{c}}$, \\ Tieyi Zhang ${ }^{1, d}$, Wenzheng Jiang ${ }^{2, e}$ and Chi Zhang ${ }^{1, f}$ \\ ${ }^{1}$ School of .Mechanical Engineering, Guangxi University, Nanning 530004, China \\ ${ }^{2}$ Guangxi Transportation Research Institute, Nanning 530007, China \\ a1514236866@qq.com ,b754205880@qq.com, ㄷ46557560@qq.com, \\ dzty1zty2@163.com , e 14913978@qq.com ‘ 2804033674@qq.com
}

Keywords: piezoelectric quartz sensor, weigh-in-motion , singular spectrum analysis

Abstract: Factors that affect the measuring accuracy of highway Weigh-in-Motion systems are analyzed and the hardware of a new Weigh-in-Motion system are analyzed and designed, then the measuring data of the Weigh-in-Motion system is processed. Finally, the method of Singular Spectrum Analysis are applied to the final calculation of vehicle weight, and the accuracy of final results is improved. The research results are helpful to highway transportation system to protect road from damage.

\section{Introduction}

The random vehicle vibration, impact load during movement and other external factors increase the complexity of vehicle movement, and at same time bring many problems to the dynamic weighing of vehicles. The main factors that affect vehicle dynamic weighing accuracy are as follows[1]:

Vehicle Vibration: The reasons that cause vehicle vibration are variable. The most important reason is from the vehicle itself, such as: engine vibration caused by eccentric rotation of periodic, periodic vibration caused by the tire tread, vibration caused by uneven combustion of oil, random vibration and uneven vibrations caused by the driver's unstable manipulation.

Road Roughness or Irregularity: Vehicle vibration caused by forced vibration forms, such as the road surface roughness caused by vibration. When the wheel contact with the road, the pavement roughness of the high frequency components has little impact on the automotive vibration due to the elasticity of the tire, thus low frequency signal plays a leading role in the vibration of an automobile.

Vehicle Speed: Random load increase with increasing speed of vehicle and curse the ground because the vibration of the vehicle combination increases, so speed is a factor affecting the dynamic precision. When the vehicle accelerate or decelerate, the elastic element will store or release some energy, the vehicle itself produces vibrations or shocks will be accompanied by two types of interference in movement[2],.

So the appropriate load cells need to be chosen and designed to reduce or eliminate the interference of the weighing platform structure, and select the appropriate sampling amplifier signal amplification process, and SSA algorithm is taken to calculate the final vehicle weight, which will ultimately control results within a predetermined error. 


\section{Dynamic Weighing System Hardware Design}

Choice of Weighing Sensor The weighing sensor of dynamic weighing systems, mainly are the following[3]: Curved plate sensor, copolymer compound piezoelectric sensors, capacitive sensors, bridge weighing platforms, fiber optic sensors, integral digital intelligent weighing sensors, piezoelectric quartz sensors [4]. Piezoelectric quartz sensor drift is extremely small, In low-speed, high-speed state, it can be used to weight, while the physical properties of quartz is relatively stable, the sensitivity changes little with time. Quartz crystal has the ability to credit-sensitive components load on the entire surface. The dynamic accuracy of weighing system can be improved when piezoelectric quartz sensor is selected as a dynamic weighing system load weighing sensor [5].

The structure of a piezoelectric quartz load cell is shown in Fig.1 and Fig.2 is for the crystal weighing body of the WIM sensors with the overall package. Quartz WIM sensors can be buried in the cement and asphalt concrete pavement in accordance with the cross-sectional dimensions shown in Fig.3. In a variety of road installation including the bridge the road is cut to $7 \mathrm{~cm}$ wide mounting groove, the depth of excavation is only $5 \mathrm{~cm}$.

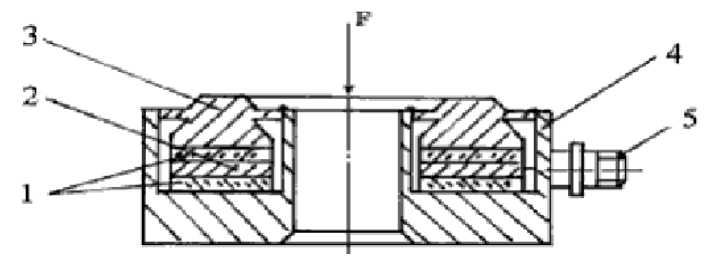

1. Quartz sheet 2.Electrode plates 3.Platen 4.Outer casing 5.Signal input socket

Fig. 1 Sectional view of the piezoelectric quartz load cell structure

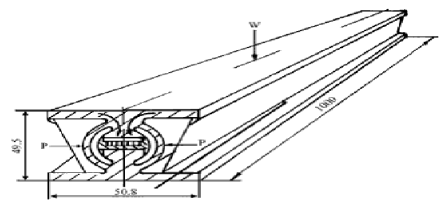

Fig.2 Piezoelectric quartz crystal weighing body

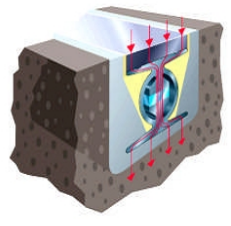

Fig.3 Sensors embedded in the road surface

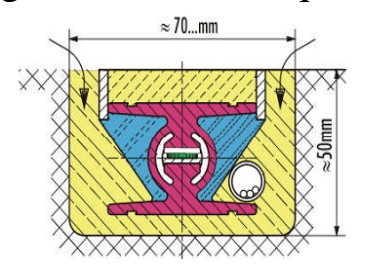

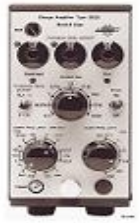

Fig. 4 The charge amplifier

Charge amplifier means supporting the quartz crystal sensor, as shown in Fig. 4. A / D converter changes the amplitude of the continuous analog signals into discrete digital signal and sends them to the computer processing. Computer receives A / D signal feed, and finish signal storage and processing.

Hardware circuit Dynamic weighing system hardware design mainly includes: Piezoelectric quartz sensor, amplifier module, a filter circuit modules, analog / digital (A / D) converter module, TMS320C6416 signal processing module, memory module, PCI interface module, a power supply circuit module, its constituent blocks is shown in Fig.5. In accordance with the system hardware design requirements and design ideas, according to the system hardware design shown in the case of hardware circuit 


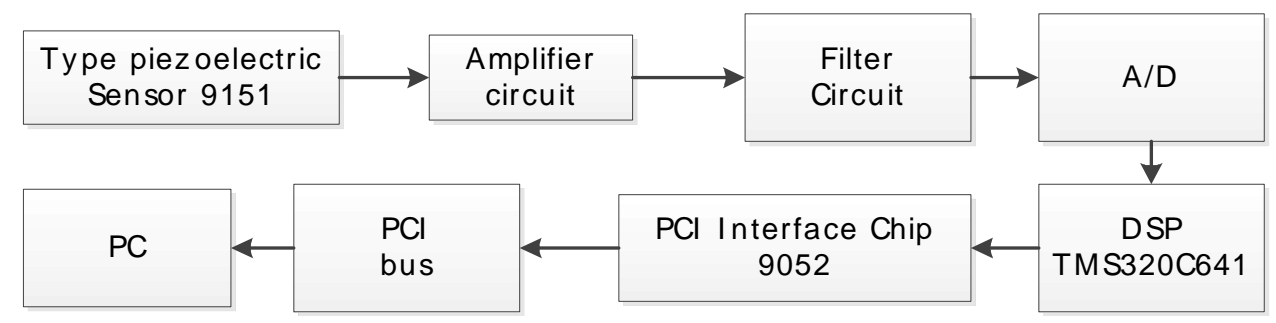

Fig.5 System hardware diagram

\section{Dynamic Weighing System Software Design}

Vehicle dynamic weighing system software design mainly includes: the main program design, data collection procedures, data processing, dynamic data fusion, display program, the communications procedures.

Software is written by Delphi, the real-time data is stored in a text file, and then call and text files in real-time data, and the results in order to query the database. data storage management subsystem for processing the collected data results after storage management to prepare for subsequent calls and inquiries. The weighing charges subsystem uses the measured data to calculate correlation algorithm based on the total weight of the vehicle, the system interface can directly display gross vehicle weight, and by entering the fees, the cost required to pay is real-time displayed, data contained in bills as a print content is displayed on its interface.

\section{Data Processing Algorithms}

SSA Algorithm The SSA algorithm is the singular spectrum analysis (abbreviated as SSA) algorithm for signal acquisition processing. The data acquisition processing is as shown in Fig.6. The sequence implied waveform signal extracted from the noise out of the system, choose the appropriate empirical orthogonal function and principal components to reconstruct the signal. For optimum noise reduction effect, the most important thing is to choose the best reconstruction order p. This is as shown in Fig.7. Using SSA algorithm for data processing can meet the design requirements to ensure high accuracy requirements.

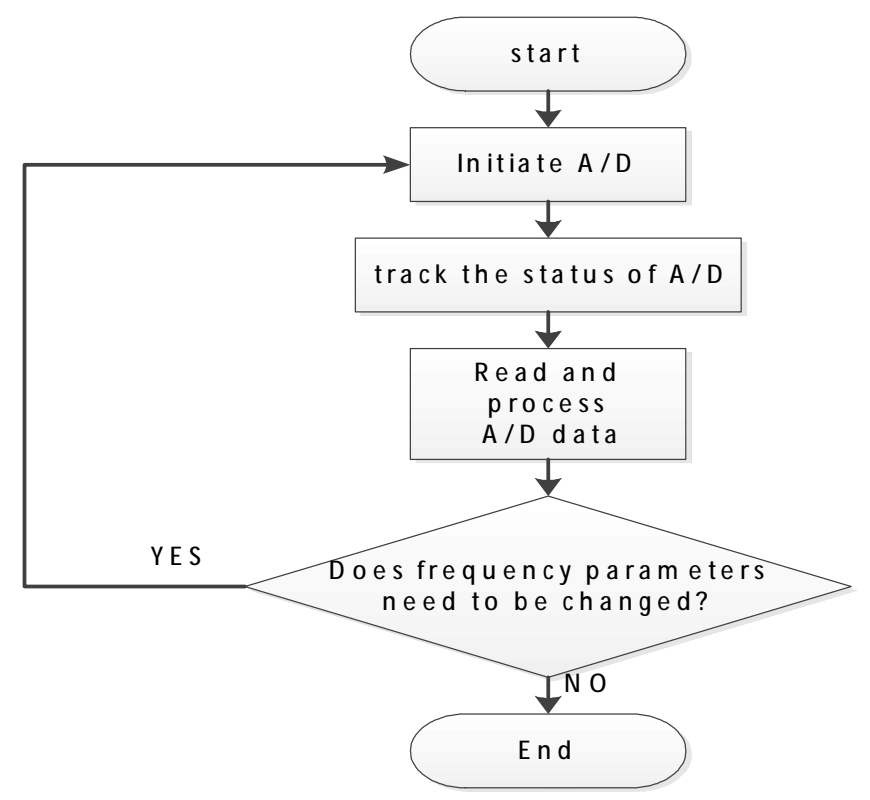

Fig.6 Data acquisition subsystem program flowchart 


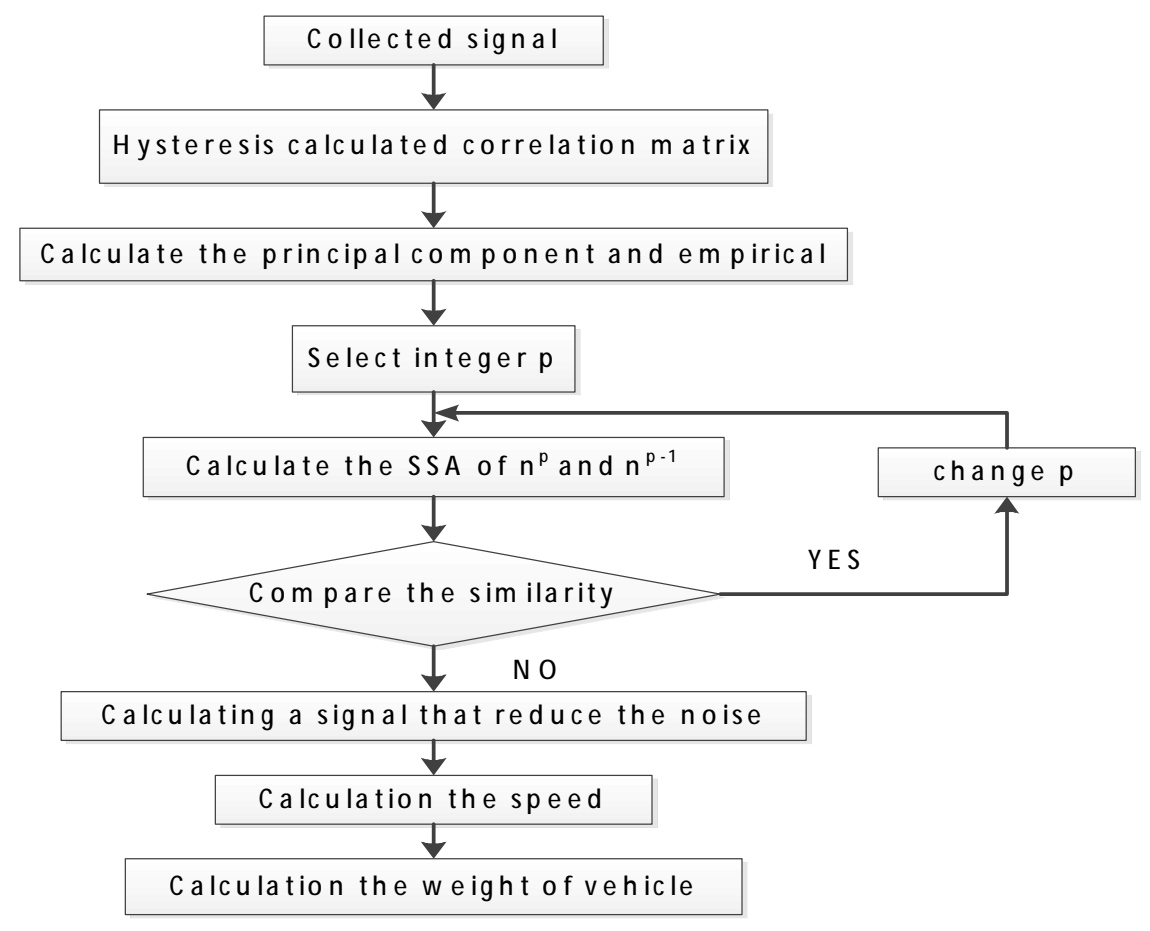

Fig.7 SSA algorithm flow chart

\section{Conclusion}

The piezoelectric quartz sensor weighing sensor principle and superiority and the characteristics of the system itself are analyzed in this paper. Secondly, the appropriate sensor and its appropriate package and the piezoelectric quartz sensor amplifiers is selected, A / D converter is chosen to meet the requirements. Finally, the design and selection of dynamic weighing system software platform which use a data processing method SSA algorithm for a piezoelectric quartz sensors that is really to maximize the final measurement results.

\section{Acknowledgment}

The research is supported by Guangxi Key Project of Science and Technology (Granted No.135500810) and supported by Guangxi Key Laboratory of Manufacturing System \& Advanced Manufacturing Technology, The research is carried out with Guangxi Transportation Research Institute and Guangxi Nanning Jiesai Digital Technical Company.

\section{Reference}

[1]LiXiao-bao,Jian Shui-zhen,Peng Hui-xiang.Piezoelectric quartz weighing sensr in the application of highway dynamic weighing[J].Highways\&automotive .Applications, 2012. (2):92-94.

[2]Wu Qisheng, Wang Dan and other research system axle load data processing algorithms [J]. ETC vehicle dynamic weighing Highway and Transportation Research, 2009 (10): 190-194

[3]Zhang Wen bin , Wang Qi , Ma Songlin2, Li Xin.kai. A Novel Multisensor System for Moving Vehicle Classification[J]. Journal of Tianjin University. 2008(41):194-198.

[4]Zheng Jian-xia. Infra.red intelligent vehicle classification counter[ J] . Journal of Chang.an University : Natural Science Edition,2003, 23(3) : 85-87

[5]Hu Fang-ming, Jian Qin, Zhang Xiu-jun. The classifier of vehicle types using BP neural networks [J] . Journal of Xidian University,2005, 32(3):439-442 